\title{
Projection de l'autre et construction de soi : modalités de l'ethos diaristique dans quelques journaux d'écrivains québécois des années 1980
}

\author{
Manon Auger \\ Université du Québec à Montréal
}

Le journal intime est la volière des écrivains. La boite à captures. Mais quelle déception d'y découvrir parfois, au milieu des beaux oiseaux rares, quelque hérisson tout en pointes, ou quelque pie-grièche en mal de radotage, ou même, certains jours, quelque gros paon vaniteux qui prend toute la place avec sa roue en éventail.

Louise Maheux-Forcier 
De par sa nature de texte écrit, tout journal intime, peu importe ses prétentions au secret, s'inscrit d'emblée sous un mode communicationnel et, se faisant, implique une relation à l'Autre, ne serait-ce que dans cet autre qu'est le journal lui-même. Cependant, lorsque le journal est dès le départ destiné à une publication, comme c'est parfois le cas pour certains journaux d'écrivain, cet Autre se dédouble dans le spectre du lecteur à venir qui, peut-on supposer, ira au journal afin de satisfaire davantage un intérêt pour la figure de l'écrivain mis en scène, qu'un intérêt pour le genre diaristique lui-même. Dès lors, l'ambiguïté du contrat proposé à l'écrivain - s'adonner à un genre intime de façon publique - conduit ce dernier en terrain glissant, pris qu'il est entre l'investissement révélateur qu'exige l'écriture diaristique et la nécessité de préserver, voire de construire une figure d'écrivain recevable tant pour lui-même que pour le lecteur projeté.

Dans cet article, c'est précisément la mise en place et les modalités de ce que nous pourrions nommer un «ethos diaristique ", propre à l'écriture des journaux d'écrivains commandés par un tiers et destinés à une publication immédiate, que nous souhaitons explorer. Plus particulièrement, nous étudierons l'implication de la figure du lecteur dans l'écriture de ce type de journaux et évaluerons les diverses possibilités formelles permettant aux écrivains de «faire œuvre» à travers (ou malgré) cette pratique, cela à partir de l'exemple de textes québécois publiés dans les années 1980. Dans l'histoire du journal intime québécois, cette décennie marque de fait un tournant important en ce qui concerne l'institutionnalisation du genre, puisque, à cette époque, plus d'une dizaine d'œuvres diaristiques paraissent chez divers éditeurs, entre autres à cause de l'initiative de Jean- 
Guy Pilon qui propose à plusieurs auteurs québécois - Yves Beauchemin, Nicole Brossard, Louise Maheux-Forcier, Michèle Mailhot et Madeleine Ouellette-Michalska - de participer à une série radiophonique intitulée " Journal intime de... » et diffusée sur les ondes de Radio-Canada. À ces journaux d'écrivain « en série », publiés par la suite par différentes maisons d'édition, s'ajoutent les deux tomes du journal de Claude Jasmin, Pour tout vous dire (1988), commandé par l'éditeur Guérin, et Pour ne rien vous cacher, qui paraît chez Leméac en 1989, ainsi que le Journal de mille jours d'André Carpentier (Guérin,1988), journal d'écriture rédigé dans le cadre d'une thèse en création menée à l'université de Sherbrooke et qui répond lui aussi bien que d'une façon moins directe - à des impératifs de commande et de publication ${ }^{1}$. Formant un corpus relativement homogène à la fois par leur proximité historique et leurs visées pragmatiques, les journaux de cette période posent également, chacun à sa manière, la question de l'inscription du public dans l'écriture dite intime et se déploient à travers diverses stratégies de séduction, de légitimation et de subversion ainsi qu'une volonté de faire œuvre qui manifestent un ethos propre à ce type d'écriture, articulé sur une volonté plus ou moins affirmée d'assurer la cohésion de la figure de l'écrivain.

\footnotetext{
1 Plusieurs autres journaux paraissent pendant cette décennie, mais nous ne les retenons pas pour différentes raisons: soit il ne s'agit pas de journaux commandés ou encore il ne s'agit pas de journaux de personnes engagées dans une carrière d'écrivain. Dans la décennie 1980, sont publiés notamment les journaux d'Élaine Audet (1989), de Marc Chabot (1988), de Gil Courtemanche (1989), d'Henriette Dessaulles (1989), de Lionel Groulx (1984), de Jean-Pierre Guay (1986-1990), de Françoise Hamel-Beaudoin (1988), de Laurent Laplante (1988) et de Jean-Louis Major (1984).
} 


\section{Tenir un journal pour être lu}

Écrire un journal, même dans l'intimité la plus stricte, ne va pas nécessairement de soi, car, s'adonner à l'exercice diaristique, c'est affirmer du même coup son droit à la singularité et, surtout, son droit à la parole ${ }^{2}$. Tenir un journal destiné à la publication, c'est, de surcroît, affirmer son désir de donner à lire la part intime de soi et, par conséquent, de partager une expérience individuelle qui non seulement n'est pas réorganisée à la manière d'une autobiographie ou d'un roman mais qui, de plus, est non exemplaire étant donné que le diariste, en écrivant au jour le jour, ne peut mesurer la valeur de l'expérience consignée qui se dessine presque à son insu. En d'autres termes, écrire un journal pour publication, du moins dans l'esprit de la majorité des écrivains-diaristes du corpus étudié, revient à la fois à transgresser les lois d'une pratique traditionnellement réservée à la sphère privée et à s'adonner à un genre qui s'édicte sur des principes fort différents de ceux des genres canoniques. Cela n'est pas sans créer chez eux un énorme malaise, comme le reconnaît entre autres André Carpentier :

Tenir un journal et accepter par avance l'idée de sa publication dénote chez le scripteur un certain fond d'orgueil; ne faut-il pas en effet considérer sa propre image, sa pensée, son langage dignes de la mémoire des êtres de demain pour risquer son énergie et sa sensibilité dans une entreprise aussi exigeante, aussi périlleuse? (p. 303)

\footnotetext{
2 Comme le souligne Mirna Velcic-Canivez, tous les écrits autobiographiques sont peu ou prou marqués par la recherche et/ou l'affirmation d'une légitimité de dire : "Pour pouvoir raconter sa vie en partie ou comme une histoire individuelle et obtenir d'être écouté, il faut avoir une place parmi les locuteurs "privilégiés" » (p. 246).
} 
Les écrivains-diaristes en sont immanquablement conscients, les dangers qui guettent celui qui s'adonne à une telle pratique sont multiples: Carpentier trouve malaisé le « dosage d'exigences et de limitations sociales et personnelles » (p.38) qu'implique cette écriture publique, Louise MaheuxForcier, souligne les dangers de la " pose », de l' « artifice » et du «mensonge» (p.17), Michèle Mailhot admet avoir de la «difficulté à soulever [...] longtemps sa vie au-dessus de l'insignifiance » (1986, p. 165), alors qu'Yves Beauchemin redoute plus que tout «l'autoglorification en sourdine, le maquillage des états d'âme et le discours pour la postérité » (p.121). À ces réticences que partagent presque tous les diaristes, s'ajoutent également la crainte de blesser un membre de l'entourage ${ }^{3}$, la possibilité de produire malgré soi une fiction des événements ${ }^{4}$ et la peur d'ennuyer les lecteurs à force de futilités ${ }^{5}$. Le "péril » et «l'exigence» (Carpentier, p. 303) de cette « entreprise redoutable» (Beauchemin, p. 102) trouvent toutefois leur exutoire privilégié à travers ces nombreux commentaires sur la pratique diaristique et les problèmes éthiques et poétiques qu'elle soulève, commentaires qui envahissent littéralement l'espace textuel, revenant régulièrement dans chaque journal comme autant de refrains

\footnotetext{
3 « Moi et ma peur de me faire de la peine et d'en faire aux autres! » (MaheuxForcier, p. 194)

4 « Ces carnets, on se demande s'ils n'ont pas peu à peu organisé en leurs pages le fléchissement de l'événementiel au seul profit d'une réflexion - lâche, aveugle - sur le scriptuaire [sic] ; comme si tu voulais, en apparence, t'y retirer presque l'usage de la personne sociale. » (Carpentier, p. 200).

5 « Coq-à-l'âne fréquents en rédigeant un journal? Ça me plaît. Vague inquiétude... Est-ce que ça va plaire à un assez grand nombre pour que je puisse persévérer dans ma nouvelle entreprise? » (Jasmin, 1988, p. 57).
} 
qui rythment l'œuvre, parfois au point de la transformer en journal du journal.

En somme, le journal public, malgré l'envie qu'on en a, fait peur, et ce n'est qu'avec bien_des appréhensions, des bémols et des questionnements que les écrivains s'y engagent; sous leur regard, il semble en effet « miné d'invisibles explosifs » (Mailhot, 1986, p. 9). Sous le regard du lecteur, par contre, le journal commandé à un écrivain est plutôt rempli de sentiments de gêne et d'inhibition ainsi que d'accès de modestie. Et cette rhétorique, qui sert d'armature au récit, permet déjà de produire $\mathrm{du}$ texte et d'en programmer la réception problématique, tout en faisant écran avec la personne de l'écrivain. Autrement dit, le journal destiné à la publication apparaît comme un difficile exercice pour qui s'y trouve confronté. Pas étonnant, dans ces conditions, que cela tourne au supplice pour certains : "Qu'est-ce que vous me voulez au juste? s'exaspère Nicole Brossard. De la littérature qui n'en aurait pas l'air? De l'écriture qui n'en serait pas? Do you want me to look cute? » (p. 48) Certes, la question se pose, mais, derrière ces innombrables justifications qui parasitent la communication, les diaristes se cachent et se protègent de l'œil supposé trop curieux du lecteur, mais ne peuvent cacher leur malaise, l'extraordinaire tension qui les fait avancer à tâtons dans ce genre, avec mille précautions qui détruisent par avance la spontanéité inhérente à l'écriture diaristique.

Si plusieurs de ces écrivains-diaristes ne parviennent jamais à surmonter véritablement leur malaise face au futur lecteur, mais s'y plient bon gré mal gré, Madeleine OuelletteMichalska et Claude Jasmin préfèrent, quant à eux, renverser la vapeur en plaçant ce dernier aux premières loges de leur 
journal, écrivant ouvertement pour lui et faisant de lui leur narrataire officiel ${ }^{6}$. Ce n'est pas tant, faut-il le préciser, qu'ils s'estiment plus dignes d'intérêt que les autres, mais plutôt qu'ils s'appuient sur le principe que l'expérience individuelle, aussi banale ou extraordinaire soit-elle, reconduit, du même coup, l'expérience commune. Ainsi, pour Madeleine-OuelletteMichalska :

La matière du journal intime concerne donc moins ce qui lie l'auteur au je du récit, que la densité du rapport qui s'établit à la lecture entre deux expériences, deux attentes, deux imaginaires. Ainsi, ce journal est intime, non pas parce que l'impudeur ou l'indiscrétion aurait pu m'inciter à vous raconter des détails de ma vie, mais parce que vous acceptez de le recevoir. (p. 161)

C'est aussi cette volonté de créer un sentiment d'empathie et une communion d'expérience que valorise Claude Jasmin :

Je cherche, ici comme dans d'autres genres d'ouvrages, à être clair, simple, attrayant aussi. Je me répète que je ne suis guère différent des autres personnes humaines, que nous avons tous, à divers degrés peut-être, les mêmes soifs, les mêmes désirs, les mêmes angoisses existentielles et les mêmes frustrations. Alors je fonce, naïf et confiant à la fois. (1989, p. 406)

De l'intérêt du public dépend même le sort de son journal; si le public n'est pas preneur dès la parution du premier tome,

\footnotetext{
${ }^{6}$ Un exemple parmi d'autres chez Jasmin : « Demain dimanche, tenez, j'en planterai encore deux ou trois [des sapins] en pensant à vous, lecteurs hypothétiques penchés sur mon passé. » (Jasmin, p. 73). Ouellette-Michalska : «Le journal intime est mémoire et fidélité. Je suis là parce que vous vous $y$ trouvez. J'écris parce que quelqu'un lira. Que ces fragments témoignent d'événements exceptionnels ou d'un quotidien banal reste secondaire. Tout est dans l'intensité du rêve. Tout est dans le désir partagé. » (OuelletteMichalska, p. 47)
} 
Jasmin avertit qu'il cessera l'exercice 7 , même si cela devait entraîner un sentiment de déception. Pour lui, en effet, le «certain petit public» du journal constitue un lectorat privilégié, « une grosse famille. Sorte de clan. De tribu. » (1988, p.17) Si cette figure du lecteur n'est pas aussi fortement intégrée à la trame textuelle du Sommet d'un arbre (Yves Beauchemin), la volonté d'établir une communication d'égal à égal avec les lecteurs constitue toutefois un rempart nécessaire pour éviter de tomber dans les nombreux pièges tendus par le journal public :

Comment discourir sur soi pendant cinq demi-heures sans se mettre à nu? Il n'y a qu'une sortie de secours : le bavardage. Il faut avoir, je pense, le courage de ne pas s'y précipiter en se rappelant avec modestie que tous les hommes et toutes les femmes partagent les mêmes joies et les mêmes misères. C'est ce qui explique peut-être qu'on puisse intéresser parfois quelqu'un par ses livres. (p. 104-105)

Cela n'empêche bien sûr pas le contact « de l'auteur et du lecteur» d'être «rude et direct» (p.104), mais il semble qu'accepter cette transgression de la règle «fondamentale » du journal - soit accepter la présence du lecteur - permette à ces trois diaristes d'être plus à l'aise dans ce «jeu de société évolué » que constitue le journal de commande (Beauchemin p. 103). Mais sur ce chapitre, il faut rester prudent, car, derrière cette apparente soumission, se cache encore le désir de plaire, de créer des affinités, de parvenir à composer avec un public restreint qui est, par le fait même, plus exigeant. Bref, de projeter une image qui rendra le diariste plus sympathique au lecteur.

\footnotetext{
7 « Bien, ça se peut qu'après un premier tirage de ce journal, l'éditeur me dise “Stop! Ça n'intéresse personne mon pauvre vieux!” Eh! Je stopperai ou, le pli étant pris, je le tiendrai pour moi tout seul. J'aime tant ça.» (Jasmin, p. 117)
} 


\section{Tenir un journal ou faire œuvre?}

Dans ces conditions, comment parvenir à faire œuvre malgré tout? Car c'est, on peut le supposer, ce qui est attendu de ces diaristes, puisque c'est leur statut d'écrivain qui leur confère non seulement le droit à la parole diaristique, mais aussi et surtout, le droit à une large diffusion de cette parole jugée d'emblée littéraire et donc digne d'intérêt. Au delà de la gênante mais à la fois désirée présence du lecteur, il apparaît que le genre diaristique lui-même, par sa forme infiniment souple et le contenu biographique qu'il présuppose, représente également un double défi. En effet, il est, d'une part, le lieu d'une possible remise en cause du statut d'écrivain de celui qui le tient et, d'autre part, sa forme, au contraire de celle des genres canoniques, semble l'éloigner de l'œuvre littéraire proprement dite ${ }^{8}$. Comment, dès lors, l'écrivain pourrait-il, à travers ce genre dévalorisé, non seulement faire la preuve de son talent, mais aussi parvenir à produire un écrit digne d'intérêt? À la question «À qui raconter?», dont la réponse éthiquement

\footnotetext{
${ }^{8}$ Depuis fort longtemps, en effet, le journal est considéré comme un genre mineur et facile, dont la banalité n'a d'égale que celle du quotidien et de la personnalité du sujet qui le tient. Cette perception très négative du genre a surtout été véhiculée par le discours critique et théorique français sur le journal. Pour voir ou comprendre ce phénomène, on pourra se référer, entre autres, à Madelénat (p. 1217), Dufief (p. 108) et Lejeune et Bogaert (p. 34-37) Pour notre part, nous jugeons que ces définitions reposent en grande partie sur des critiques positivistes et biographiques dont les fondements théoriques ont depuis longtemps été dépassés. Cependant, si la recherche actuelle permet de revoir cette définition pour le moins délicate, elle n'en demeure pas moins fortement ancrée dans la culture littéraire ambiante, tout particulièrement celle des années 1980. D’ailleurs, André Carpentier et Claude Jasmin reconduisent, chacun dans leur journal, la vision de la pratique diaristique à cette période, en inscrivant les commentaires négatifs qu'ils entendent sur le journal : Voir : Carpentier (p. 36 et p. 62-63) et Jasmin (p. 36)
} 
problématique ne peut être esquivée par les écrivains-diaristes, s'ajoutent dès lors les questions «Que raconter? » et surtout " Comment le raconter? ». Celles-ci, à leur tour, vont constituer à la fois la matière et la manière du journal et contribuer à la mise en place de principes et de valeurs qui modèleront l'image de soi véhiculée par le journal.

\section{La réécriture}

Afin d'assurer l'intérêt de leur œuvre, la réécriture ${ }^{9}$ apparaît comme une option privilégiée par plusieurs diaristes de notre corpus. Elle peut prendre des formes très diverses. Par exemple, dans l'esprit d'André Carpentier, qui n'en est alors qu'au début de sa carrière ${ }^{10}$, légitimer la publication de son journal implique une réécriture régie par la résolution « d'expurger le Journal, d'éliminer [...] son contenu autobiographique» (p. 292). Pour parvenir à un tel résultat, cependant, Carpentier s'est d'abord laissé une certaine marge de manœuvre dans l'écriture des jours afin de procéder ensuite à l'intellectualisation du contenu de son journal, par le bannissement presque total du moindre détail quotidien ou sentiment personnel : «Ce Journal comptera

\footnotetext{
9 Il faut cependant entendre ce terme dans tous les sens, soit autant la coupe, la censure, le polissage ou encore "toute reprise d'une œuvre antérieure, quelle qu'elle soit, par un texte qui l'imite, la transforme, s'y réfère, explicitement ou implicitement, d'une manière qui n'est pas un simple commentaire ou une copie, mais une appropriation ou un détournement. » (Bordas, p. 501)

${ }_{10} \mathrm{Au}$ moment où il commence la rédaction de son journal en 1983, il a déjà publié cinq titres, mais le plus important de sa production paraîtra après cette période. Notons également qu'il est le plus jeune des diaristes de notre corpus, puisqu'il a entre 36 et 39 ans lors de la rédaction de son journal, alors que les autres sont dans la quarantaine ou la cinquantaine.
} 
moins, j'espère, par les quelques témoignages biographiques qu'il contiendra - il sera nettoyé de ses scories - que par son esthétique, sa textualité, et la pensée qui l'accompagnera. » (p. 129) En d'autres termes, il s'agit pour lui de désincarner son propos afin de le faire accéder à une pensée plus universelle et, donc, plus légitime. Journal d'écrivain parce que journal d'écriture, sonJournal de mille jours est ainsi l'exemple parfait du journal autoréflexif qui érige sa matière en réfléchissant sur son propre fonctionnement et ne laisse qu'une place toute relative $\mathrm{au}$ « je » du scripteur. Ce n'est donc pas tant le statut d'écrivain de Carpentier qui est mis en relief dans ce journal que sa formation d'écrivain.

Faire œuvre à travers - ou malgré - la forme d'un journal emprunte aussi chez Madeleine Ouellette-Michalska dans La tentation de dire, journal, la voie de la réécriture du manuscrit original. Dans son cas, il s'agit non pas d'en rendre plus impersonnel le contenu, mais d'assurer un certain lissage du récit diaristique. En effet, le journal qu'elle livre à l'édition est une version considérablement remaniée de textes lus sur les ondes de Radio-Canada ${ }^{11}$. Dans ce journal -identifié ainsi à cause de la mention générique « journal » qui fait office de soustitre -, les déictiques, indicateurs de la situation d'énonciation de la diariste et caractéristique essentielle de la forme diaristique, ont tous ou presque été gommés afin de donner une œuvre aux repères temporels pour le moins incertains mais au fini beaucoup plus concerté. Ce choix poétique, outre qu'il

\footnotetext{
11 « De la voix à l'écriture. Ce livre a eu comme point de départ une diffusion à Radio-Canada FM dans la série "Journal intime" de l'été 84. Par la suite, il y eut des ajouts, des remaniements, des transformations. Les textes d'origine se sont ouverts en de nombreux cahiers dont je n'avais prévu ni l'aboutissement ni l'orientation. » (p. 9)
} 
traduit un désir évident de faire œuvre malgré la forme fragmentée du journal, permet aussi de faire passer au premier plan l'esthétique de l'essai autobiographique qui vient ainsi supplanter celle du journal. À son tour, cette esthétique permet de donner préséance aux événements significatifs de la carrière de l'écrivain pendant l'année où les textes ont été écrits, ce qui ajoute à la crédibilité et à la notoriété de la diariste - qui se tient en respect derrière l'écrivain - et permet d'assurer la cohérence du message général véhiculé par La tentation de dire, soit une triple valorisation : de la littérature québécoise, de la littérature des femmes et de la langue française.

Alors que Ouellette-Michalska accepte de bonne grâce la présence du public dans son journal, Michèle Mailhot, qui tient depuis longtemps un journal intime, se sent quant à elle soudainement gênée dans son écriture dès qu'il s'agit de transformer sa pratique en une écriture de commande :

Jamais je n'aurais pensé que la rédaction de ce journal que j'ai toujours écrit si allègrement au coulant de la plume, sans aucune rature, ne devienne tout à coup si difficile du seul fait qu'il risque de ne plus être intime. Déjà, toutes les barrières, empêchements et difficultés de la création se sont dressés entre lui et moi. (1984, p. 15)

Elle se livre ainsi au défi de Jean-Guy Pilon avec une grande humilité. Sentant dans cette commande non pas une consécration de son statut d'écrivain, mais la nécessité de faire œuvre, de répondre à une certaine exigence, elle opte elle aussi pour une réécriture qui se dessine à l'intérieur même de son parcours d'écriture. Ainsi, $L a$ vie arrachée ${ }^{12}$, rédigé en 1984 , est tout entier

\footnotetext{
12 Michèle Mailhot produit en fait deux textes pour la série « Journal intime de... » : La vie arrachée (1984) et Notes de parcours (1986). Dans le présent
} 
construit sur une intertextualité avec des cahiers antérieurs de la diariste, cahiers qui coïncident avec le deuil de ses deux fils et dont elle reprend des extraits dans la trame même de son nouveau journal: "Pour les fins de ce travail, explique-t-elle, j'ai donc sorti [l]es quarante-trois cahiers [de mon journal] remplis d'une écriture serrée, rendue à peu près illisible pour les autres dans les passages les plus délicats. » (1984, p. 9) Si Mailhot ne précise pas ce qui l'a conduite à choisir ces extraits précis parmi les cahiers de son journal - ni si certains de ces «passages les plus délicats » sont désormais lisibles -, nous voyons toutefois se dessiner dans l'ensemble que constitue $L a$ vie arrachée l'aboutissement d'un processus de deuil important pour la diariste, processus qui se redessine à travers l'itinéraire du journal, mais dont il constitue aussi une part importante puisque, par la volonté de partager une expérience traumatisante, la diariste semble se livrer à une sorte de délivrance. À cet égard, la forme diaristique paraît être celle qui convient le mieux à ce cheminement car il ne s'agit pas de revivre les événements que Mailhot répugne manifestement à révéler, mais bien plutôt de placer au cœur de l'écrit la perception qu'elle en a, les sentiments et le trouble qu'ils ont suscités et, par la même occasion, de transformer en une œuvre cohérente des événements qui relèvent nécessairement de la brutalité de l'existence. L'exercice diaristique public devient donc, pour Mailhot, à la fois l'occasion de dire et de ne pas dire, mais aussi l'occasion de faire œuvre et d'assurer, par la

article, nous nous en tiendrons à l'étude du premier segment du journal que constitue La vie arrachée et qui forme un tout homogène. De son côté, Notes de parcours (1986) mériterait une étude complète et autonome, distincte de la première, car on constate, tant dans la question de l'ethos que dans la matière et la manière du journal, une certaine rupture avec la première œuvre, en dépit du fait que cinq mois seulement les séparent. 
coïncidence des événements rapportés qui découle de cette réécriture, la cohérence du récit diaristique qui représente en lui-même la continuation d'un important processus de deuil.

\section{L'intergénéricité}

Nous venons de le voir, Carpentier, Mailhot et OuelletteMichalska, en exposant leur capacité de faire œuvre à partir de leurs journaux, proposent - et cela davantage de biais que de front - une image publique d'eux-mêmes qui les pose en écrivains. Pour y parvenir, toutefois, ils ont d'abord dû se livrer assez librement à l'exercice diaristique avant de pouvoir adopter la posture de l'écrivain qui livre au public un journal intime et qui propose, du même coup, dans le contenu ou dans la forme adoptée par leur nouveau journal, un écrit dans lequel ils se sentent plus à l'aise. Par contre, pour Louise MaheuxForcier dans Le sablier et Nicole Brossard dans Journal intime ou voilà donc un manuscrit, le rapport au genre diaristique et la nécessité de faire œuvre par le journal se posent d'une manière beaucoup plus conflictuelle; sans doute, et c'est là notre hypothèse, parce qu'elles adoptent d'emblée - c'est-à-dire dans l'écriture des jours - une posture stricte d'écrivain qui sert en quelque sorte de rempart entre le lecteur et elles, mais surtout entre le genre diaristique et elles. Destinant ouvertement leur journal à un public qui connaît et apprécie déjà leurs œuvres, c'est de front qu'elles se mesurent à eux.

Chez Louise Maheux-Forcier, habituée à tenir un journal intime, l'écriture coule assez librement; mais, pour elle comme pour bien d'autres, il s'agit tout de même d'en dire le moins 
possible avec le plus de mots possibles ${ }^{13}$. Ainsi, ses réticences, ses justifications et ses réflexions sur le journal occupent déjà une bonne part de l'œuvre, part à laquelle viennent s'ajouter des réflexions diverses sur des thèmes propres aux journaux d'écrivains : l'écriture, la langue, les œuvres, les voyages, etc. Au niveau formel, Maheux-Forcier intègre à l'ensemble diaristique des fragments autobiographiques - qui sont souvent, dans tous les journaux d'écrivains, l'occasion de redessiner son passé à la lumière de sa formation d'écrivain -, ainsi que des poèmes, des rêves, des impressions de voyage et privilégie particulièrement les fragments, les poèmes en prose, les pensées et les aphorismes, toutes formes qui excluent sensiblement le sujet biographique. On retrouve donc, dans Le sablier, plusieurs pages de notations générales et relativement désincarnées qui servent d'écran entre la diariste et le lecteur, tout en attestant de cette capacité de l'écrivain à faire du général à partir de l'expérience singulière, de transformer le narcissiqueje en

\footnotetext{
13 Ce cheminement est tout de même tortueux chez Louise Maheux-Forcier et est reconduit à l'intérieur même du journal. Cette écrivaine, qui refuse de mener une vie publique où elle se sent mal à l'aise, accepte tout de même le contrat du journal parce que, comme il s'agit d'écriture, cela touche plus directement à son art et parce qu'elle espère que le journal permettra de donner une image plus juste d'elle-même, voire de justifier certaines de ses prises de position. Cependant, elle réalise vite qu'il s'agit d'un jeu dangereux à bien des niveaux : «Après avoir tant de fois tenu bon en refusant de paraître à la télévision ou de parler à la radio, après avoir tant de fois dit "non" à tous ces réalisateurs et interviewers que j'estime, au risque de les blesser et de m'attirer de fâcheuses épithètes, je reconnais avoir accepté cette invitation-ci avec enthousiasme pour la belle raison qu'elle excluait ma personne, sans réfléchir suffisamment aux écueils qui attendaient mon stylo, lequel n'aura même pas pour excuse, s'il se conduit mal, la terreur des micros et la phobie des caméras. C'est vraiment tout à fait extraordinaire d'être aussi étourdie à mon âge et de n'avoir perçu d'abord, dans ce projet de journal radiophonique, que des échos de paradis intime... » (Maheux-Forcier, p. 16) Son journal est dès lors dominé par la crainte de se dévoiler, crainte toutefois assez bien cachée sous un masque de fermeté et d'affirmation de soi.
} 
un nous plus généreux. Certaines de ces notations et de ces aphorismes, d'ailleurs, sont l'occasion de réfléchir - et de renseigner du même coup le lecteur - sur la condition d'écrivain ${ }^{14}$. De plus, même si l'auteur semble prendre plaisir à l'entreprise diaristique au point d'ironiser fréquemment à son sujet - arguant qu'il faut que ses finances «soient en piteux états » pour qu'elle ait accepté d'embarquer " dans cette galère du "journal intime" » (p. 109) -, sa crainte de se révéler la mène, tout comme Brossard, au bord d'une exaspération qui repousse le lecteur aux frontières du journal :

Je viens de me jouer un sale tour! D'abord, j'ai cru que c'était une bonne idée, car, enfin! quelle idée de croire qu'un journal radiophonique puisse être un véritable journal intime! Il y a des intimités qui ne passent pas la rampe! et, depuis quelques jours, je me faisais des misères avec certains paragraphes qui dépassaient les bornes! "Quelles bornes?" Ça me regarde! moi et la décence! (p. 194)

Cette résistance, qui touche ici davantage au contenu du journal, menace toujours d'exploser à la face des écrivainsdiaristes et prend, chez Nicole Brossard, une forme plus extrême encore qui se matérialise en une réticence complète vis-à-vis du genre diaristique lui-même. Cependant, son malaise ne vient pas tant, comme chez les autres, du fait que le journal doit être livré au public, mais émane plutôt de sa conception du genre qui serait, selon ses termes, le lieu par excellence de

\footnotetext{
14 Les exemples sont nombreux : «On a tort de croire que les écrivains sont plus attentifs que les autres et qu'ils sont doués d'un sens de l'observation plus aigu. Bien au contraire, un écrivain, c'est quelqu'un qui vit en perpétuel état de distraction et qu'un mot suffit à replonger dans son œuvre de la même façon qu'une odeur ramène un cheval où l'on sait! au grand galop! » (p. 66) «Que voulez-vous! même si les écrivains sont reconnus pour être de fieffés menteurs, il est des cas où ils ne résistent pas aux vérités qui dépassent la fiction! » (p. 94)
} 
« l'enlisement du sujet » (p. 33). C'est donc à rebours qu'elle s'y engage, répugnant à parler d'elle-même mais surtout à le faire à travers un journal. Toute son entreprise est dès lors axée sur une volonté de s'inscrire en faux par rapport à une tradition du journal - mais on est en droit de se demander laquelle -, de poser son œuvre dans les marges de cette pratique qu'elle interroge tout au long de son parcours pour finalement avouer que «le journal ne [lui] suffit pas. Ne [lui] convient pas»: «C'est une forme d'écriture qui exige trop de moi et pas assez de ce que je suis. » (p. 65) Un peu paradoxalement, son refus du genre permettra malgré tout de produire un texte, par ailleurs un des plus courts de tout le corpus.

C'est principalement la crainte de mettre à mal la figure d'écrivain de Brossard qui semble présider à ce refus du genre diaristique, mais cette crainte est d'abord compensée par un réseau intergénérique important. Par exemple, comme, pour Brossard, la poésie est la forme suprême qui s'oppose le plus radicalement à la banalité du journal, elle prend soin, après chaque partie de journal, d'ajouter ce qu'elle appelle des "postures du texte et un poème »; " sans doute, explique-t-elle, pour que rien ne m'échappe et que tout puisse commencer » (p. 10, souligné dans le texte). Les postures servent ainsi de " passage » entre le journal et le poème, elles permettent à la fois de transcender le «rien» du journal afin d'accéder au «tout » de la poésie et attestent, du même coup, de la valeur de la diariste en tant que poète, capable de transformer le quotidien en littérature. Ensuite, pour celle qui affirme que « aussi réelle que possible, [s]a vie n'est qu'un tissu de mots » (p.13), l'inscription du biographique est, autant que faire se peut, bannie du journal pour laisser toute la place au langage, à l'écriture et à l'exercice de style. Toutefois, afin de tisser une 
trame narrative minimale, mais, surtout, afin d'utiliser les rares éléments biographiques à son avantage, Brossard opte pour un jeu de constructions semi-fictionnelles fait de superpositions d'entrées qui auraient été écrites à des moments marquants de sa vie. Rien ne nous confirme si ces entrées inscrites dans la trame temporelle de 1983 et datées de 1961, 1970 et 1973 jusqu'à 1982 ont réellement été tirées de cahiers antérieurs, mais il est plus probable qu'il s'agit d'une posture autobiographique adaptée à la forme du journal et adoptée dans le but de se substituer à l'éreintant exercice quotidien, d'autant plus que ces entrées reprennent des moments-clés du parcours d'écrivain de Brossard et permettent de la mettre en scène en tant que féministe intellectuelle fortement engagée et reconnue. En somme, la posture autobiographique, au même titre que la présence de poèmes, semble fournir des repères à l'écrivain et empêcher, dans la mesure du possible, de remettre en question un statut pourtant fragilisé par une volonté si prégnante de l'affirmer et par le refus obstiné de l'auteur de s'adonner à la forme diaristique.

\section{Le compromis}

Dans cette galerie de journaux d'écrivains, le journal d'Yves Beauchemin, Du Sommet d'un arbre, occupe une position médiane. Fruit d'une première diffusion à l'émission « Journal intime de... » sur les ondes de Radio-Canada, il est constitué de deux segments (février - mai 1983 / février - avril 1985) et précédé de deux textes autobiographiques, «Enfance» et « La ville », tous deux rédigés pour d'autres séries radiophoniques. Présentés ici non pas dans l'ordre chronologique de leur 
rédaction, mais dans celui des éléments autobiographiques qu'ils mettent en scène (l'enfance, le passage à la vie adulte symbolisé par le déménagement à la ville, suivi des journaux qui relatent des moments dans la vie de l'écrivain), ces textes instaurent ainsi un mode de lecture à la fois marqué par l'intergénéricité (particulièrement avec le genre de l'autobiographie) et par la réécriture (le réaménagement des divers fragments, mais aussi leur agencement et leur très plausible «polissage »).

Mettant en place certains éléments autobiographiques qui introduisent la figure du diariste dans une forme d'écriture plus convenue institutionnellement mais qui autorise la subjectivité, la réflexion et l'organisation narrative, les textes proprement autobiographiques de $\mathrm{Du}$ Sommet d'un arbresoulignons que la désignation générique de cette œuvre est « journal » - préparent en quelque sorte le terrain à la venue de l'écriture diaristique. Retraçant la période qui va de l'enfance abitibienne à l'âge adulte montréalais, ils conduisent le lecteur au seuil du journal qui, d'ailleurs, s'ouvre sur un fragment autobiographique tiré une nouvelle fois de l'enfance du diariste et rattaché fort habilement, à la fin de l'entrée, à l'événement du jour qui l'a fait naître; le lecteur - tout comme l'auteur - bascule ainsi d'un mode d'énonciation à l'autre sans le moindre heurt et cela tout au long des premières entrées du journal qui allient diarisme, autobiographie, essai et prose romanesque. L'écriture diaristique avance donc assez plaisamment chez Beauchemin, sans la présence de ces stratégies d'évitement que l'on a pu voir chez les autres diaristes et sans que cette pratique ne semble poser à son auteur de problèmes éthiques ou esthétiques particuliers. Cependant, cette sérénité apparente se révèle bientôt n'être qu'un masque prêt à se fissurer; d'abord 
doucement, dans une remarque équivoque ("Fondamentalement inutile, cette autopsychanalyse pour diffusion publique », p.93), puis, de façon éclatante, dans une longue entrée entièrement métadiscursive qui vient, selon les dires mêmes de l'auteur, "briser l'illusion » référentielle du journal. Beauchemin laisse alors tomber ses défenses et avoue humblement ses craintes: «Mais, comme la plupart des écrivains qui ont participé à cette série, je me suis rapidement pris au jeu et maintenant je le trouve un peu... compromettant. Comment parler de soi durant deux heures et demie sans devenir vulnérable?» (p. 103) Conscient de cette vulnérabilité et des pièges qui se dressent devant lui, Beauchemin comprend toutefois que l'évacuation de son individualité ne serait qu'une voie de contournement qui diminuerait singulièrement l'intérêt de son œuvre :

En écrivant ce texte-ci, où j'essaie de lutter contre une force d'attraction qui cherche à m'amener dans des endroits où je n'aimerais pas aller quand je suis seul, je continue de me trahir, de laisser deviner mes peurs, mes méfiances, mes phobies. Je ne sais plus trop quel écrivain disait que la voie royale de la littérature passe par l'inconscient. Ceux qui parviennent à l'éviter (ou qui n'arrivent pas à l'emprunter) tournent autour $\mathrm{du}$ pot et ennuient leurs lecteurs, car on se lasse autant d'un homme qui ne parle jamais de lui-même que de celui qui en parle tout le temps. (p. 104, je souligne)

Une fois ces questions soulevées - et en partie résolues presque immédiatement -, Beauchemin reprend le fil de l'écriture diaristique là où il l'avait laissé, sans se perdre dans les fauxfuyants ou les commentaires métadiscursifs - sauf lorsqu'il entreprendra le deuxième journal, de manière à poser d'emblée les balises du contrat qu'il signe avec ses lecteurs. Il se propose ainsi de jouer franchement le jeu diaristique, c'est-à-dire qu'il 
accepte de se mettre directement en scène, sans fausse pudeur mais sans excès, et témoigne de sa capacité à faire de l'universel à partir de son expérience singulière, sans pour autant oblitérer cette dernière. Malgré le caractère «faux et artificiel» de ce « charmant casse-gueule appelé Journal intime et radiophonique » (p.121), il ne s'agit pas pour le diariste de s'en tenir scrupuleusement aux règles du genre diaristique, mais, plutôt, en tant qu'écrivain, de s'adonner à une expérience d'écriture bâtie sur la résurgence de plusieurs thématiques qui lui sont chères (l'enfance, la musique, la politique, l'écriture, la ville, etc.) dans un langage qui lui est familier, celui du romancier (plusieurs entrées sont composées sous forme de mini-récits avec dialogues). De plus, même s'il est conscient d'avoir un statut particulier en tant qu'écrivain - ses deux premiers romans, L'Enfirouapé et Le Matou ayant connu un succès populaire important - au point parfois de «subir quelque chose qui ressemble à un début de mythification» (p.100), Beauchemin ne cherche pas, à travers cette pratique, à cautionner son autorité en tant qu'écrivain ; pour lui, il s'agit simplement d'un métier, d'une fonction tout aussi importante que celles de père de famille ou de citoyen du Québec. La question du rapport problématique au genre du diarisme public se pose donc chez Beauchemin d'une façon beaucoup plus ciblée et se résout également à l'intérieur même de l'œuvre, l'auteur parvenant à trouver, par le biais de la réécriture et de l'intergénéricité, un certain équilibre entre la révélation de soi et la nécessité de faire œuvre.

\section{Conclusion}

Le journal intime, cela semble indéniable, " est la volière des écrivains. La boite à captures », pour emprunter l'expression de 
Maheux-Forcier (p. 290). Mais si les lecteurs veulent admirer ces beaux grands oiseaux que sont les écrivains, mieux vaut, semble-t-il, les observer en liberté... Car, de fait, écrire un journal pour les autres sur commande, dans une forme fragmentée et en parlant de soi, est un contrat pour le moins malaisé à mener à bien. Mais alors que certains - on pense ici à Jasmin, à Carpentier, à Mailhot et à Beauchemin - surmontent malgré tout leur malaise pour se livrer à l'exercice, sinon avec bonheur du moins avec aisance, d'autres - on pense ici à Brossard et à Maheux-Forcier - ne parviennent jamais à vaincre leur résistance au genre et se sentent continuellement menacés par cette forme d'écriture. Outre que cela manifeste, d'une certaine manière, la complexité du genre, on peut voir dans ces nombreux jeux de cache-cache auxquels se livrent tous les journaux de ce corpus une façon de construire une figure d'écrivain qui va de la modestie la plus feinte à l'affirmation la plus agressive.

Bien sûr, il faut se méfier de toute schématisation, mais nos exemples semblent vouloir confirmer l'hypothèse que, moins le diariste cherche à assumer une autorité forte en tant qu'écrivain, plus il se sent à l'aise dans la pratique diaristique, alors que, inversement, plus le diariste a une conscience élevée de son statut, plus sa résistance au genre est grande. Loin de nous toutefois l'idée de vouloir valoriser une poétique ou une autre ou encore de vouloir dénoncer la prétention des uns et l'humilité des autres - sentiments qui sont toujours très relatifs et coincés dans l'ethos problématique du journal. Il est en revanche frappant de constater que ceux qui ne se sentent autorisés à produire du journal que sous leur condition d'écrivain se sentent du coup obligés de travailler la forme, de l'adapter, de la travestir afin d'assurer leur crédibilité en tant 
qu'auteur. Inversement, ceux qui intègrent le statut d'écrivain à un éventail de conditions plus large - tels, chez Jasmin, celui du conjoint, du père, du grand-père, du feuilletoniste, du polémiste; ou encore, chez Mailhot, celui de la mère endeuillée, de la femme séparée; ou chez Beauchemin, celui du père, de l'homme, du citadin, etc. - se sentent plus à l'aise pour parler d'euxmêmes et se glissent avec un certain confort dans la posture du diariste. Par exemple, chez Claude Jasmin, la surconscience du public-lecteur construit la figure d'un écrivain sympathique, engagé surtout à livrer ce qu'on attend de lui et affirmant par ailleurs avec une grande modestie son statut d'écrivain. Son attitude est donc radicalement opposée à celle de Nicole Brossard ou de Louise Maheux-Forcier qui parviennent à ne pas se trahir en se dissimulant derrière la barrière du langage et en adoptant un discours fortement marqué par l'intergénéricité. Cela ne donne pas lieu, ni d'un côté ni de l'autre, à des œuvres d'une plus grande qualité esthétique, car la pudeur y est toujours présente, tout autant que la crainte du lecteur, le danger de la pose et de la banalité du quotidien, mais ces questions peuvent alors se jouer sur un autre terrain, celui du contenu.

À ce niveau, on remarque à quel point le choix de la matière textuelle est complexe : le biographique peut se voir évacué au profit de généralités désincarnées (Carpentier, Maheux-Forcier), le quotidien peut être supplanté par la recréation rétrospective d'événements significatifs (OuelletteMichalska, Brossard), la fragmentation du journal gommée par un récit organisateur (Ouellette-Michalska, Mailhot), etc. De plus, les réflexions métadiscursives omniprésentes sont comme autant de preuves que l'écrivain réfléchit sur son art, tandis que les craintes et les réticences freinent le mouvement de l'écriture 
et de la lecture tout en produisant du texte; les faits sont souvent cachés ou oblitérés pour ne laisser que quelques traces écrites qui ne s'enracinent plus dans l'expérience individuelle, mais dans l'expérience générale et le hors temps. Pas étonnant, dans ces conditions, que quelques-uns de ces écrivains-diaristes se soient fait dire que, même après la diffusion ou la lecture de leur journal, on n'en savait pas plus sur eux ${ }^{15}$. C'est qu'il faut, pour cela, parvenir à lire entre les lignes et étudier comment la façon de se raconter véhicule davantage de sens que le propos lui-même (comme le remarque Beauchemin: " On se trahit d'ailleurs surtout par le ton», p. 103). Il faut aussi repérer comment le genre diaristique infléchit une certaine image de soi, conditionnée par la présence de l'Autre qui observe et qui juge, mais qu'il s'agit d'amadouer, de séduire, de convaincre. Une tension s'installe donc à l'intérieur du journal d'écrivain destiné à la publication par cette interaction entre un locuteur et son lecteur projeté qui détermine presque entièrement la posture adaptée par le premier. Cependant, tout porte à croire

\footnotetext{
15 Pour preuve, ce commentaire de Louise Maheux-Forcier : «Après la lecture de mon journal de l'an dernier, cet ami nouveau que j'aime comme un ami d'enfance m'avait écrit une merveilleuse lettre, avec la conclusion suivante : “Et pourtant, je ne sais toujours pas qui vous êtes!" Hélas! moi non plus! Tout ce que je sais, c'est que j'ai reçu au berceau, en double partage, le talent d'écrire et le talent d'aimer... et que j'essaie depuis lors d'en faire bon usage, en tâchant d'oublier que la perfection n'est pas de ce monde et que l'œuvre humaine est toujours un "devenir interrompu". » (p. 206). Même observation chez Claude Jasmin après sa lecture des quatre premiers tomes du journal de Jean-Pierre Guay (1986-1990) : "J'ai dû lire mille pages de ses confessions jusqu'ici et je ne sais pas du tout encore qui il est. L'impression d'un secrétaire perpétuel de ses téléphones et lettres personnelles. Rien que ça. » (Jasmin, 1988, p. 418). Par contre, le journal de Claude Jasmin crée exactement l'effet inverse : «Je constate la différence entre publier un roman et publier un tome de journal. Bien des gens me parlent de cette récente publication, toujours avec une sorte de regard excité, une complicité, l'impression sans doute qu'ils sont entrés chez moi un peu par effraction. » $(1989$, p. 311)
} 
que, à travers ce corpus propre à un contexte géographique et à une période de l'histoire du journal, nous nous trouvons en face d'une version exacerbée de l'ethos qu'implique toute écriture de l'intime.

\section{Bibliographie}

BEAUCHEMIN, Yves. 2001 [1986]), Du sommet d'un arbre, journal [1983-1985], Montréal, Bibliothèque Québécoise.

BORDAS, Éric. 2002, «Récriture, Réécriture », dans Paul Aron, Alain Viala et Denis Saint-Jacques (dir.), Le dictionnaire du littéraire, Paris, Presses universitaires de France.

BRosSARD, Nicole. 1984, Journal intime, ou, Voilà donc un manuscrit, Montréal, Les Herbes Rouges.

CARPENTIER, André. 1988, Journal de mille jours (Carnets 19831986), Montréal, Guérin, XYZ éditeur.

DuFIEF, Pierre-Jean. 2001, Les écritures de l'intime de 1800 à 1914 : autobiographies, mémoires, journaux intimes et correspondances, Rosny-sous-Bois, Bréal.

GuAY, Jean-Pierre. 1986-1990, Journal (1985-1988), Montréal, Pierre Tisseyre, 6 volumes.

JASMIN, Claude. 1988, Pour tout vous dire, Montréal, Guérin.

—. (1989), Pour ne rien vous cacher, Montréal, Leméac.

LEjEune, Philippe et Catherine Bogaert. 2006, Le journal intime. Histoire et anthologie, Paris, Textuel. 
MADELÉNAT, Daniel. 1996, "Journal intime », dans Beaumarchais, Couty, Rey (dir.),Dictionnaire des littératures de langue française, Paris, Bordas, volume 2, p. 1217-1220.

MAHEUX-FORCIER, Louise. 1984, Le sablier. Journal intime 19811984, Montréal, Pierre Tisseyre.

Mailhot, Michèle. 1984, La Vie arrachée. Cahiers [Journal, 1977/1978/1983], Montréal, La Presse.

—. (1986), Notes de parcours [Journal, 1983-1984], Montréal, La Presse.

Ouellette-MichalskA, Madeleine. 1985, La tentation de dire. Journal, Montréal, Québec/Amérique.

VelCiC-CANiveZ, Mirna. 1997, « Le pacte autobiographique et le destinataire », Poétique, no 110, p. 239-254.

\section{Résumé}

À partir de l'exemple de quelques journaux d'écrivains québécois commandés et publiés dans les années 1980, cet article interroge la mise en place et les modalités d'un " ethos diaristique » propre à ces œuvres impliquant, dans leur dynamique communicationnelle, une relation problématique au lecteur. Trois composantes majeures de cet ethos sont plus particulièrement examinées : les difficultés à discourir sur soi, la nécessité de faire œuvre à travers ou en dépit de la forme du journal et le désir de préserver, voire de construire une figure d'écrivain recevable tant pour le diariste que pour son lecteur projeté. 


\begin{abstract}
Based on a number of diaries written in the eighties by Québec writers at the request of their publishers, this paper examines the modalities of a "diaristic ethos" typical of these works, showing the way they relate, sometimes problematically, to the reader. Three major aspects of this ethos are examined: the difficulty of speaking about oneself, the necessity of writing a literary work by way of - or against - the diary format, and the desire to preserve or perhaps even to construct a writer's persona that is acceptable for the diarist as well as for the intended reader.
\end{abstract}

